\title{
Encefalopatía perdedora de sal en romboencefalitis por listeria
}

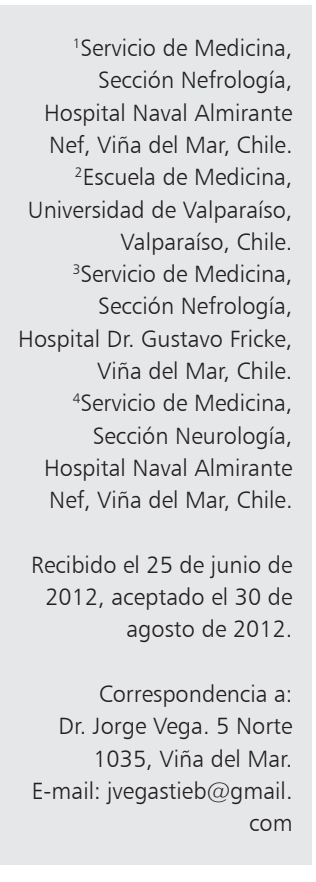

\author{
JORGE VEGA $^{1,2,3}$, GONZALO MATAMALA ${ }^{4}$ \\ Cerebral salt wasting syndrome associated \\ with Listeria monocytogenes encephalitis. \\ Report of one case
}

\begin{abstract}
Hyponatremia is common in patients with severe neurological diseases and is often secondary to a syndrome of inappropriate antidiuretic hormone secretion (SIADH). However, in some patients, hyponatremia is due to cerebral salt wasting syndrome (CSWS). SIADH and CSWS treatments are opposite and misdiagnosis can lead to increased morbidity and mortality. We report a 52 years old female with a rhomboencephalitis caused by Listeria Monocytogenes (LM), ventriculitis and abscesses in cerebellum and brainstem. It was associated with hyponatremia, hypotension, increased natriuresis, hypouricemia, and low creatinine and blood urea nitrogen levels. Large amounts of sodium were needed and the condition persisted after hospital discharge. Hyponatremia is common in central nervous system involvement by LM, however we are not aware of CSWS reports of this condition.
\end{abstract}

(Rev Med Chile 2013; 141: 104-108).

Key words: Hyponatremia; Listeria monocytogenes; Natriuretic peptides.
L a hiponatremia $(\mathrm{HNa})$ es una complicación frecuente en patologías neurológicas graves $^{1}$. Pocos años después de ser descrita, la encefalopatía perdedora de sal (EPS) pasó al olvido luego de publicarse el primer reporte del síndrome de secreción inadecuada de hormona antidiurética (SIADH) ${ }^{2,3}$. Posterior al año 1981 esta entidad volvió a recibir atención al identificarse, en diversas series, casos de hiponatremia asociados a hipovolemia especialmente en pacientes neuroquirúrgicos ${ }^{4,5}$.

Estas dos causas de HNa presentan muchas características en común. Se suele diagnosticar un SIADH si el volumen extracelular es normal o algo elevado, hay una osmolalidad plasmática baja y menor a la urinaria, sodio urinario $>20 \mathrm{mEq} / \mathrm{L}$, hipouricemia, hormona antidiurética $(\mathrm{ADH})$ elevada con bajas concentraciones de renina y aldosterona. Sin embargo, en algunos pacientes la $\mathrm{HNa}$ se debe a una pérdida de sal y la terapia habitual del SIADH puede agravar su condición aumentando la morbilidad y la mortalidad ${ }^{6}$.
Comunicamos el caso de una paciente con una romboencefalitis por listeria monocitogenes que presentó una EPS.

\section{Caso clínico}

Mujer de 52 años con antecedentes de infección urinaria recurrente y asma bronquial por lo que utilizaba intermitentemente corticoides. Ingresó al hospital afebril (bajo antipiréticos), con presión arterial 90/60mmHg, pulso 94, luego de 2 días de dolor lumbar, fiebre y cefalea. Presentaba contractura muscular paravertebral y dolor que se exacerbaba con la puño percusión. El examen de orina mostró leucocitos: $5-10 \mathrm{p} / \mathrm{c}$, piocitos, hematíes: 1-3 $\mathrm{p} / \mathrm{c}$, nitritos $(+)$, bacterias +++ . El hematocrito fue 36,5\%, leucocitos: $12.500 \mathrm{~mm}^{3}$, plaquetas: 244.000 $\mathrm{mm}^{3}$, proteína C reactiva: $44,2 \mathrm{mg} / \mathrm{L}(\mathrm{VN}:<4,9)$. La ecotomografía renal fue normal. Se diagnosticó una pielonefritis aguda e indicó ceftriaxona $2 \mathrm{~g} /$ día. Al tercer día, por persistir la fiebre y vómitos se 
agregaron amikacina y ondasentrón. Al cuarto día el urocultivo mostró una E. coli multisensible. La cefalea motivó un scanner de cerebro que no mostró alteraciones. Al sexto día amaneció en sopor, el líquido cefalorraquídeo mostró hipoglucorraquia $(9 \mathrm{mg} / \mathrm{dl})$, proteínorraquia $(360 \mathrm{mg} / \mathrm{dl})$ y pleocitosis $\left(18 \mathrm{~mm}^{3}\right)$, VDRL $(-)$, adenosin-deaminasa (-) y latex (-) para neumococo, meningococo y hemophilus. Se aumentó la ceftriaxona a 4 g/día y se agregaron dexametasona, vancomicina $1 \mathrm{~g}$ $\mathrm{c} / 12 \mathrm{~h}$ y aciclovir endovenoso. Después de unas horas mejoró el nivel de conciencia y se constató diplopía en la visión lateral, nistagmus central, dismetría bilateral e hiperreflexia generalizada. La clínica y la tinción de Gram de LCR con bacilos Gram (+) hicieron plantear una romboencefalitis por listeria e iniciar ampicilina ( $8 \mathrm{~g} / \mathrm{d}$ dí). La fiebre cesó ese día y en los siguientes aparecieron paresia del velo del paladar, leve hemiparesia derecha y un temblor de acción en las extremidades superiores. La resonancia magnética mostró hiperintensidad en secuencias T2 y FLAIR en región pontobulbar, cerebelo y pedúnculos cerebelosos, al igual que en el sistema ventricular, en particular en el tercer y cuarto ventrículo, con difusa captación de Gadolinio en $\mathrm{T} 1$ en las mismas zonas. Al confirmarse bacteriológicamente una romboencefalitis por listeria monocitógenes y descartarse otras etiologías, se suspendió el aciclovir, vancomicina y ceftriaxona.

Al día 13 del tratamiento, una nueva punción lumbar y resonancia magnética mostraron persistencia de la hipoglucorraquia y pleocitosis de predominio poliformonuclear, consolidación de los microabscesos en el tronco y mayor captación de gadolinio en pedúnculos cerebelosos, lo que llevó a prolongar la terapia antibiótica.

Estando recibiendo entre 600 y $1.300 \mathrm{ml}$ de solución salina diarios, el día 20 apareció poliuria de $6.150 \mathrm{ml} /$ día asociada a hiponatremia $(132,9$ $\mathrm{mEq} / \mathrm{L})$, natriuresis de $506 \mathrm{mEq} /$ día, fracción excretada de sodio elevada (3,29\%), hipotensión arterial con ausencia de taquicardia y función renal normal. Se sospechó una disfunción del sistema nervioso autónomo y una EPS, aumentándose el aporte de sodio oral y endovenoso. Tres días después se midió la concentración del péptido natriurético cerebral (BNP) que resultó normal. En las semanas siguientes, mejoró su estado neurológico pero continuó con poliuria, natriuresis superior al aporte, hipotensión arterial, uricemia baja y tendencia a la hiponatremia (Tabla 1). La terapia antibiótica se suspendió al mes (21 días con ampicilina). Fue dada de alta a los 36 días del ingreso, persistiendo sólo la diplopía en la mirada hacia arriba. Los exámenes previos al alta mostraron hiponatremia leve $(133,5 \mathrm{mEq} / \mathrm{L})$, uricemia baja (2,9 mg/dl), fracción excretada de ácido úrico discretamente elevada $(11,2 \%-\mathrm{VN}:<10 \%)$, hipernatriuresis (772 mEq/día) con fracción excretada de sodio alta $(4,75 \%)$ y función renal normal (uremia: $17 \mathrm{mg} / \mathrm{dl}$, creatinina: 0,84 mg/ $\mathrm{dl}$, clearance de creatinina: $104 \mathrm{ml} / \mathrm{min} / 1,73 \mathrm{~m}^{2}$ ).

En los controles ambulatorios posteriores se constató que su manejo renal del sodio y la presión arterial se normalizaron sólo cinco semanas más tarde y que no quedaron secuelas neurológicas. Posteriormente, recordó haber ingerido queso de cabra artesanal en la semana previa al inicio de su enfermedad, lo que pudo haber sido la fuente de contagio.

\section{Discusión}

La hiponatremia es frecuente en pacientes con meningitis bacteriana ${ }^{7-9}$. En un estudio efectuado en 671 adultos, $30 \%$ de ellos tenían hiponatremia a la admisión. Fue más frecuente en los enfermos con infección por listeria $(73 \%)$ que en las por neumococo $(33 \%)$ y meningococo $(21 \%)^{7}$. La etiología de la hiponatremia en las meningitis no es clara y puede deberse a un SIADH, aporte excesivo de líquidos o EPS ${ }^{10,11}$.

EPS es definida como una pérdida renal de sodio durante una enfermedad intracraneal que lleva a hiponatremia, natriuresis excesiva y depleción de volumen. La hiponatremia revierte con volumen y sal ${ }^{12}$. La poliuria es frecuente ${ }^{9,13}$. Desgraciadamente los hallazgos de laboratorio en SIADH y EPS son iguales y la depleción de volumen no es fácil de reconocer clínicamente, dificultando su diferenciación con SIADH. Debe prestarse atención a la presencia de cambios ortostáticos en la presión arterial y el pulso, venas yugulares planas, mucosas secas, ascensos en la proteinemia y el hematocrito, pérdida de peso y al balance de fluidos en las hojas de enfermería ${ }^{1}$. Si se considera apropiado puede estimarse el estado de la volemia con procedimientos invasivos o radioisotópicos ${ }^{14}$.

La EPS se ha descrito en pacientes con meningitis, traumatismos craneanos, tumores cerebrales, hemorragia sub-aracnoídea, encefalitis, tubercu- 
Tabla 1. Parámetros previos y durante el período de poliuria en una paciente con romboencefalitis por Listeria monocitogenes y encefalopatía perdedora de sal

\begin{tabular}{|c|c|c|c|c|c|c|c|}
\hline Día de hospitalización & 15 & $19 *$ & 20 & 22 & 26 & $34 * *$ & $\begin{array}{l}14 \text { días } \\
\text { post alta }\end{array}$ \\
\hline Sodio plasmático (mEq/L) & 132,9 & & 132,9 & 137,9 & 135,4 & 133,5 & 141 \\
\hline Cloro plasmático (mEq/L) & 100,1 & & 103,8 & 107,2 & 105,9 & 105,1 & 105 \\
\hline Potasio plasmático (mEq/L) & 3,8 & & 3,5 & 3,9 & 3,3 & 3,4 & 3,8 \\
\hline Bicarbonato plasmático (mEq/L) & & & & 28,2 & & & \\
\hline Nitrógeno ureico (BUN) (mg/dl) & 10,3 & & 7,9 & 8,9 & 8,9 & 7,9 & 10,3 \\
\hline Creatinina (mg/dl) & 0,68 & & 0,67 & 0,71 & 0,70 & 0,84 & 0,78 \\
\hline Relación BUN/Creatinina & 15,1 & & 11,8 & 12,5 & 12,7 & 9,45 & 13,2 \\
\hline Uricemia (mg/dl) & & & & & 3,2 & 2,9 & 4,2 \\
\hline Aporte de $\mathrm{NaCl}$ (gramos/día) & & & 16,6 & 28,5 & 8,5 & 31 & \\
\hline Aporte de $\mathrm{NaCl}(\mathrm{mEq} / 24 \mathrm{~h})$ & & & 284 & 487,3 & 145,3 & 530,0 & \\
\hline Sodio urinario (mEq/24 h) & & & 506 & 831,0 & 412,6 & 772,5 & 172 \\
\hline Sodio urinario (mEq/L) & & & 88,8 & 123,7 & 87,8 & 107,3 & 61 \\
\hline Fracción excretada Na (\%) & & & 3,3 & 3,9 & 2,26 & 4,75 & 0,70 \\
\hline Clearance creatinina $\mathrm{ml} / \mathrm{min} / 1,73 \mathrm{~m}^{2}$ & & & 102 & 134,9 & 117,1 & 103,5 & 120,1 \\
\hline Presión sistólica máxima (mmHg) & 112 & 93 & 111 & 107 & 94 & 113 & \\
\hline Presión sistólica mínima (mmHg) & 91 & 68 & 75 & 82 & 78 & 92 & \\
\hline Presión diastólica máxima (mmHg) & 63 & 60 & 67 & 66 & 58 & 69 & \\
\hline Presión diastólica mínima (mmHg) & 58 & 43 & 49 & 52 & 51 & 56 & \\
\hline Frecuencia cardiaca máxima (LPM) & 81 & 89 & 85 & 90 & 85 & 94 & \\
\hline Frecuencia cardiaca mínima (LPM) & 58 & 78 & 72 & 71 & 76 & 80 & \\
\hline Temperatura máxima ${ }^{\circ} \mathrm{C}$ & 36,9 & 37 & 37 & 37,7 & 37,5 & 37,2 & \\
\hline Diuresis (ml/24 h) & & 6.150 & 5.700 & 6.720 & 4.700 & 7.200 & \\
\hline
\end{tabular}

*Día de inicio de la poliuria; mEq/L: miliequivalentes por litro; $\mathrm{NaCl}$ : cloruro de sodio; **último control previo al egreso hospitalario.

losis meníngea, poliomielitis bulbar, adenomas hipofisiarios, hemorragias cerebrales, carcinomatosis meníngea y cirugía in tracraneana ${ }^{1,12,15,16,17,18,19}$. También se ha comunicado en patologías extracraneanas como infecciones por HIV, enfermedad de Hodgkin, fractura de cadera y carcinomas metastásicos, razón por lo cual se ha sugerido cambiar el nombre de "cerebral salt wasting syndrome" por "renal salt wasting syndrome"14,20.

La fisiopatología propuesta para explicar la EPS es que existiría una disminución del flujo del sistema nervioso simpático (SNS) al riñón y/o una elevación de factores natriuréticos circulantes ${ }^{1,8,17}$.

La disminución del estímulo del SNS sobre el túbulo proximal (TP), que es el segmento donde se reabsorbe la mayor cantidad de sodio, produce una disminución en su reabsorción, con entrega de una gran cantidad al nefrón distal y a la orina, originando una disminución del volumen circulante efectivo. Esto último es un estímulo para los baroreceptores, aumentando la secreción de $\mathrm{ADH}$ en forma "apropiada" a la depleción de volumen a pesar de la hipo-osmolalidad, incrementando la reabsorción de agua originando hiponatremia. La disminución del tono del SNS reduce la secreción de renina y de la aldosterona. Por ello estas hormonas no se elevan a pesar de la depleción de volumen y habitualmente no se observa hipokale- 
mia, a pesar de la entrega de grandes cantidades de sodio al nefrón distal. El ácido úrico generalmente está bajo $(<4 \mathrm{mg} / \mathrm{dl})$, como también sucede en el SIADH. En SIADH se debe al VEC aumentado, en la EPS a la disminución en la reabsorción proximal por el tono simpático reducido y al aumento en la secreción tubular por la estimulación de los receptores V1 por la $\mathrm{ADH}^{18,21}$. También se ha encontrado defectos en la reabsorción de fosfatos en el túbulo proximal ${ }^{14,21}$.

Los péptidos natriuréticos auricular (ANP) y cerebral (BNP) a menudo están elevados en la EPS. Ellos tienen un efecto inhibidor en la reabsorción de sodio en el túbulo colector en la médula interna, contribuyendo a la natriuresis. También tienen un efecto inhibidor sobre la secreción de renina en las células yuxtaglomerulares, en la secreción suprarrenal de aldosterona y en el tronco cerebral, reduciendo el flujo simpático y el tono simpático al riñón ${ }^{1,22}$. El aumento del BNP es más frecuente que el de ANP en la EPS ${ }^{17,18}$.

Esta paciente presentó hipotensión arterial y ausencia de taquicardia al día 17 de su admisión, sugerentes de un trastorno autonómico originado en la inflamación del tronco cerebral. Tres días más tarde presentó poliuria ( $>6$ litros), hiponatremia (en ausencia de drogas que la produzcan) y natriuresis elevada, sin relación con las cantidades de sodio recibidas en los días previos. En los casos publicados, la aparición de EPS ocurre alrededor del día 10 de la injuria cerebral, pudiendo ocurrir después del mes 9 . A pesar de grandes aportes de sodio en las 2 semanas siguientes se mantuvo la tendencia a la hiponatremia, hipotensión arterial, ausencia de taquicardia, poliuria, hipouricemia con fracción excretada de ácido úrico (FEAU) elevada y natriuresis persistentemente superior a los aportes de sodio. Todo ello es concordante con una EPS. Este trastorno habitualmente se resuelve espontáneamente en 3 a 4 semanas ${ }^{1}$. La función renal fue normal durante toda su evolución, lo que hace improbable que la pérdida de sal haya sido secundaria a una nefropatía tubulo-intersticial por infección o por los antibióticos utilizados. La participación de los péptidos natriuréticos en su trastorno no pudo acreditarse, ya que sólo se midió la concentración BNP en una ocasión y fue normal. No se determinó el volumen circulante ni se midió osmolalidades, $\mathrm{ADH}$, renina y aldosterona.

El único parámetro capaz de diferenciar a la EPS de SIADH es la normalización de la FEAU cuando se ha corregido la hiponatremia en SIADH y la persistencia de su alteración en EPS $^{23}$. En esta paciente la FEAU persistió elevada días antes del alta pero la hiponatremia aún persistía.

Algunos autores han dudado de la existencia de la EPS. Ellos creen que los pacientes eliminan los líquidos administrados previamente por vía endovenosa durante una reanimación excesiva o excretan fisiológicamente el sodio debido a reducción de los vasos venosos de capacitancia, originada por vasoconstricción inducida por catecolaminas $^{11,24,25}$. Otro autor sugiere que, si no se puede probar si hay diferencias entre la EPS y SIADH, debe darse el mismo tratamiento: sodio. Sugiere que el nombre de "cerebral salt wasting syndrome" pudiera cambiarse por "cerebral salt wasting syndrome"26.

En suma: pensamos que comunicar este caso puede permitir a los internistas ampliar el diagnóstico diferencial de las hiponatremias en pacientes neurológicos y elegir el tratamiento apropiado para esta condición, en que la restricción de volumen y agua al paciente puede ser deletérea.

\section{Referencias}

1. Palmer BF. Hyponatraemia in a neurosurgical patient: syndrome of inappropriate antidiuretic hormone secretion versus cerebral salt wasting. Nephrol Dial Transplant 2000; 15: 262-8.

2. Peters JP, Welt LG, Sims EA, Orloff J, Needham J. A saltwasting syndrome associated with cerebral disease. Trans Assoc Am Physicians 1950; 63: 57-64.

3. Schwartz WB, Bennett W, Curelop S, Bartter FC. A syndrome of renal sodium loss and hyponatremia probably resulting from inappropriate secretion of antidiurética hormone. Am J Med 1957; 23: 529-42.

4. Nelson PB, Seif SM, Maroon JC, Robinson AG. Hyponatremia in intracranial disease: perhaps not the syndrome of inappropriate secretion of antidiurética hormone (SIADH). J Neurosurg 1981; 55: 938-41.

5. Wijdicks E. Aneurysmal subarachnoid hemorrhage. En Wijdicks E. (Ed). The clinical practice of critical care neurology. Lippincot-Raven. Philadelphia. 1997: 132-57.

6. Wijdicks EF, Vermeulen M, Hijdra A, van Gijn J. Hyponatremia and cerebral infarction in patients with ruptured intracranial aneurysm: is fluid restriction harmful. Ann Neurol 1985; 17: 137-40.

7. Brouwer MC, van de Beek D, Heckenberg SG, Spanjaard L, de Gans J. Hyponatraemia in adults with community- 
acquired bacterial meningitis. Q J Med 2007; 100: 37-40.

8. Heras Benito M, Iglesias P, Guevara P, Sánchez Hernández R, Fernández-Reyes MJ. Hyponatremia secondary to cerebral salt-wasting syndrome associated to bacterial meningitis. Nefrologia 2008; 28: 570-1.

9. Pérez Cateriano V, Lubombo Kinsay AM, Caballero Zirena AC, Alvarez Terrero A. Cerebral salt-wasting syndrome associated with bacterial meningitis. Neurología 2011; 26: 441-2.

10. Moller K, Larsen FS, Bie P, Skinhoj P. The syndrome of inappropriate secretion of antidiuretic hormone and fluid restriction in meningitis-how strong is the evidence? Scand J Infect Dis 2001; 33: 13-26.

11. Singh S, Bohn D, Carlotti AP, Cusimano M, Rutka JT, Halperin ML. Cerebral salt wasting: truths, fallacies, theories, and challenges. Crit Care Med 2002; 30: 25759.

12. Camous L, Valin N, Zagagoza JL, Bourry E, Caumes E, Deray G, et al. Hyponatraemic syndrome in a patient with tuberculosis-always the adrenals? Nephrol Dial Transplant 2008; 393-5.

13. von Bismarck P, Ankermann T, Eggert P, Claviez A, Fritsch MJ, Krause MF. Diagnosis and management of cerebral salt wasting (CSW) in children: the role of atrial natriuretic peptide (ANP) and brain natriuretic peptide (BNP). Childs Nerv Syst 2006; 22: 1275-81.

14. Bitew S, Imbriano L, Miyawaki N, Fishbane S, Maesaka JK. More on renal salt wasting without cerebral disease: response to saline infusion. Clin J Am Soc Nephrol 2009; 4: 309-15.

15. Revilla-Pacheco FR, Herrada-Pineda T, Loyo-Varela M, Modiano-Esquenazi M. Cerebral salt wasting syndrome in patients with aneurysmal subarachnoid hemorrhage. Neurol Res 2005; 27: 418-22.
16. Santana L, Hernández-Medina E, Villanueva A, SánchezPalacios M. Salt waste syndrome in subarachnoid hemorrhage. Nefrologia 2006; 26: 513.

17. Byeon JH, Yoo G. Cerebral salt wasting syndrome after calvarial remodeling in craniosynostosis. J Korean Med Sci 2005; 20: 866-9.

18. Momi J, Tang CM, Abcar AC, Kujubu DA, Sim JJ. Hyponatremia-what is cerebral salt wasting? Perm J 2010 Summer; 14: 62-5.

19. Cuadrado Godia E, Cerdá Esteve M, Rodríguez Campello A, Puig de Dou J. Cerebral saltwasting syndrome due to infectious diseases of the central nervous system. Med Clin (Barc) 2007; 128: 278-9.

20. Docci D, Cremonini AM, Nasi MT, Baldrati L, Capponcini C, Giudicissi A, et al. Hyponatraemia with natriuresis in neurosurgical patients. Nephrol Dial Transplant 2000; 1707-24.

21. Oh MS, Carroll HJ. Cerebral salt-wasting syndrome. We need better proof of its existence. Nephron 1999; 82: 110-114.

22. Levin ER, Gardner DG, Samson WK. Natriuretic peptides. N Engl J Med 1998; 339: 321-8.

23. Maesaka JK, Gupta S, Fishbane S. Cerebral salt-wasting syndrome: does it exist? Nephron 1999; 82: 100-9.

24. Carlotti AP, Bohn D, Rutka JT, Singh S, Berry WA, Sharman A, et al. A method to estimate urinary electrolyte excretion in patients at risk for developing cerebral salt wasting. J Neurosurg 2001; 95: 420-4.

25. López Gáston OD, Jorge M. Cerebral salt wasting. Half a century of largely undefined syndrome. Medicina (B Aires) 2001; 61: 890-4.

26. Sterns RH, Silver SM. Cerebral salt wasting versus SIADH: what difference? J Am Soc Nephrol 2008; 19: 194-6. 